\title{
PERIODIC RESPONSE OF MULTI-DISK ROTORS WITH BEARING CLEARANCES
}

\author{
Y. B. Kim, S. T. NOAH AND Y. S. $\mathrm{CHOI}^{\dagger}$ \\ Mechanical Engineering Department, Texas A\&M University, College Station, Texas 77843-3123, \\ U.S.A.
}

\begin{abstract}
The forced steady state response of a multi-disk rotor system involving a clearance at one of the bearings is determined by using a harmonic balance approach. The impedance method is applied to each of the harmonic steady state components in order to reduce the system to its displacement at the non-linear bearing support. The results reveal the interrelated roles of the bearing clearance, mass eccentricity and side force in producing dangerous subharmonics. The significant effects of the strong non-linearity of a bearing clearance are studied as related to the various system parameters. The results show that the approach developed in this study is computationally superior to numerical integration methods in analyzing multi-disk rotor systems with strong non-linearity.
\end{abstract}

\section{INTRODUCTION}

Several experimental studies [1-3] have demonstrated the occurrence of undesirable subsynchronous whirling of rotors interacting through a clearance with their housing or supports. The present work extends the incremental harmonic balance method developed earlier [4] for a modified Jeffcott rotor model with bearing clearances to the determination of the periodic response of non-linear multi-disk rotor systems. The significant effects of the strong non-linearity of a bearing clearance are studied as related to the various system parameters.

Several methods have recently been advanced for determining the periodic solution of single-disk rotor systems with non-linear supports [4,5-7]. For application to larger, multi-disk rotor systems, Nataraj and Nelson [8] developed a periodic solution method based on a collocation approach for the response of the rotor. They utilized a subsystem approach [9] to reduce the size of the resulting system of algebraic equations.

In general, there exist three different response types for a rigid rotor with a bearing clearance: (i) periodic response of the same period as that of the rotational speed of the rotor, (ii) sub- or supersynchronous response, and (iii) aperiodic response. To determine the response of the first or the second type, a harmonic balance method (HBM) with DFT (Discrete Fourier Transform)/IDFT (Inverse Discrete Fourier Transform) can be utilized for a multi-disk rotor with a bearing clearance, as presented in this paper. However, the third response type, which is usually more complicated than the first two [10], cannot be obtained by using this approach. A specially modified DFT/IDFT algorithm is needed to obtain this response since the frequency components are incommensurate. Such a modified algorithm is being developed separately and is not included in the present paper.

In the present work, it is shown that the harmonic balance method, as applied to a large, non-linear rotor support system, can be made to be highly efficient. This is achieved

$\dagger$ Present address: Faculty, Sung-kyun kwan University, Korea. 
by using a version of an impedance, or dynamic stiffness [11,12], in which the steady state imbalance response of a spinning rotor is described by displacements at the bearings. This is done by using a partition of the impedance matrix to relate these displacements to the imbalance forces. A similar approach was independently developed in reference [13] to reduce a set of $n$ algebraic equations, resulting from a collocation method, applied to a rotor system, to only those co-ordinates which are directly coupled to the system's non-linear components. The impedance method is applied to each of the harmonic steady state relations corresponding to each of the harmonic components of the assumed periodic solution. For illustration, the method is applied to a two-disk cantilevered rotor system with one of its bearings possessing a deadband clearance.

\section{ANALYSIS}

\subsection{EQUATIONS OF MOTION}

To demonstrate the application and efficiency of the harmonic balance method as applied to multi-degree-of-freedom finite element rotor models, a typical rotor with non-linear bearing is analyzed to determine its non-linear response to imbalance. The rotor model considered is shown in Figure 1. A bearing with deadband clearance between the outer race and the housing is located at node 2 . The equation of motion for the rotor-support system in terms of finite elements can be written as

$$
[M]\{\ddot{R}\}+[C]\{\dot{R}\}+[K]\{R\}=\{f\}+\{g(t)\},
$$

where $[M]$ is a mass matrix, including diametral moment of inertia for the disks, $[C]$ includes damping terms and gyroscopic terms, and $[K]$ is a stiffness matrix (a list of nomenclature is given in the appendix). The displacement vector $\{R\}$ is defined as

$$
\{R\}=\left\{R_{1 x}, R_{2 x}, R_{3 x}, R_{1 y}, R_{2 y}, R_{3 y}, \beta_{4 y}, \beta_{5 y}, \beta_{6 y}, \beta_{4 x}, \beta_{5 x}, \beta_{6 x}\right\}^{\mathrm{T}},
$$

where $R_{i}$ and $\beta_{i}$ denote displacement and rotation, respectively. A vector $R$ has the dimension of $12 \times 1$. The nodal displacements $7 x, 7 y, 8 x$ and $8 y$ of Figure 1 are eliminated in equation (1) because they are fixed. The forcing terms on the right side of equation (1) are those of the non-linear bearing force, $\{f\}$, and the imbalance forces, $\{g(t)\}$.
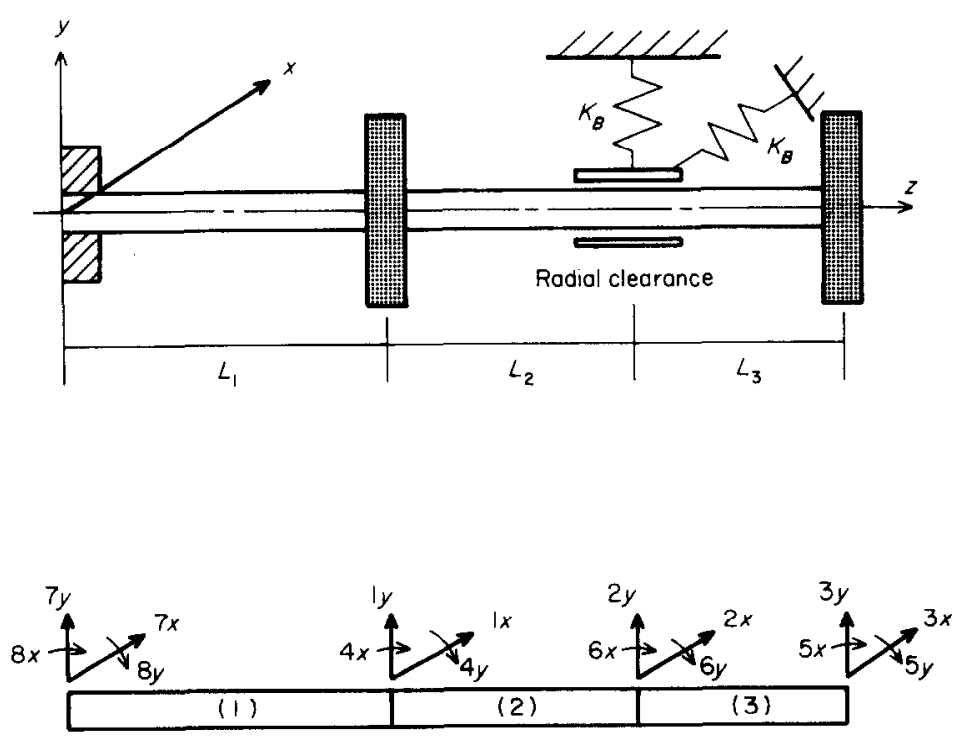

Figure 1. Finite element rotor model. 
When contact/non-contact occurs between the bearing outer race and the bearing support at node 2, the non-linear force terms can be expressed as

$$
\{f\}=\left\{0, f_{x}, 0,0, f_{y}, 0,0,0,0,0,0,0\right\}^{\top},
$$

in which [4], with $F_{s}$ as a constant side force,

$$
\begin{aligned}
& \left\{\begin{array}{l}
f_{x}=K_{B} R_{2 x}\left(1-\frac{\delta}{\sqrt{R_{2 x}^{2}+R_{2 y}^{2}}}\right)+F_{s} \\
f_{y}=K_{B} R_{2 y}\left(1-\frac{\delta}{\sqrt{R_{2 x}^{2}+R_{2 y}^{2}}}\right)
\end{array}\right\} \quad \text { if } \sqrt{R_{2 x}^{2}+R_{2 y}^{2}} \geqslant \delta \quad \text { (contact), } \\
& f_{x}=F_{s}, f_{y}=0 \quad \text { if } \sqrt{R_{2 x}^{2}+R_{2 y}^{2}}<\delta \quad \text { (no contact), }
\end{aligned}
$$

where $\delta$ denotes the bearing radial clearance and $K_{B}$ stands for the bearing support stiffness, and $f_{x}\left(f_{y}\right)$ represents the $x(y)$ direction non-linear force at node 2 . The sinusoidal excitation force vector which occurs at nodes 1 and $3,\{g(t)\}$, can be described by

$$
\{g(t)\}=\left\{g_{s}\right\} \sin \omega t+\left\{g_{c}\right\} \cos \omega t .
$$

In order to facilitate the formulation, the co-ordinates are rearranged so that the coordinates at the gap are listed first,

$$
\{R\}=\left\{R_{2 x}, R_{2 y}, R_{1 x}, R_{1 y}, R_{2 x}, \ldots, \beta_{6 y}, \beta_{6 x}\right\}^{\mathrm{T}}, \quad\{f\}=\left\{f_{x}, f_{y}, 0,0,0,0,0,0,0,0,0,0\right\}^{\mathrm{T}},
$$

in which the matrices $[M],[C]$ and $[K]$ and the force vector $\{g(t)\}$ are also changed accordingly.

\subsection{STEADY STATE SOLUTION}

The steady state, periodic solution can be assumed as follows. For the ith node,

$$
\begin{aligned}
& R_{i x}=A_{0 x}^{i}=\sum_{n=1}^{N}\left(A_{n x}^{i} \cos \frac{n \omega t}{\nu}+B_{n x}^{i} \sin \frac{n \omega t}{\nu}\right), \\
& R_{i y}=A_{0 y}^{i}+\sum_{n=1}^{N}\left(A_{n y}^{i} \cos \frac{n \omega t}{\nu}+B_{n y}^{i} \sin \frac{n \omega t}{\nu}\right), \quad i=1,2,3, \ldots, 6,
\end{aligned}
$$

where $\nu$ is the subharmonic ratio, so that

$$
\left\{\begin{array}{ll}
\nu=1, & \text { harmonic, superharmonic case } \\
\nu=\text { integer, } & \text { subharmonic case }
\end{array}\right\} .
$$

If the system reaches the steady state, the non-linear force can be expressed as [4]

$$
\begin{aligned}
& f_{x}=C_{0 x}+\sum_{n=1}^{N}\left(C_{n x} \cos \frac{n \omega t}{\nu}+D_{n x} \sin \frac{n \omega t}{\nu}\right), \\
& f_{y}=C_{0 v}+\sum_{n=1}^{N}\left(C_{n v} \cos \frac{n \omega t}{\nu}+D_{n y} \sin \frac{n \omega t}{\nu}\right) .
\end{aligned}
$$

A selection for the integer $m$ to assign to $\nu$ allows the determination of the $1 / m$ order subharmonic responses. Substituting in equation (1) from equations (3), (4) and (5) and applying the harmonic balance method result in

$$
[K]\{A\}=\{C\},
$$

where $[K]$ is a stiffness matrix and

$$
\{A\}=\left\{A_{0 x}^{2}, A_{0 y}^{2}, A_{0 x}^{1}, \ldots, A_{0 y}^{6}\right\}^{\top}, \quad\{C\}=\left\{C_{0 x}, C_{0 y}, 0, \ldots, 0\right\}^{\top} .
$$


For matrix reduction, the vectors in equation (6) can be written in the following partitioned form: for the constant term,

$$
[K]\left(\begin{array}{c}
\left\{A_{m}^{0}\right\} \\
\left\{A_{s}^{0}\right\}
\end{array}\right)=\left(\begin{array}{c}
\left\{C_{m}^{0}\right\} \\
\left\{C_{s}^{0}\right\}
\end{array}\right)
$$

where

$$
\begin{gathered}
\left\{A_{m}^{0}\right\}=\left\{A_{0 x}^{2}, A_{0 y}^{2}\right\}^{\mathrm{T}}, \quad\left\{A_{s}^{0}\right\}=\left\{A_{0 x}^{1}, A_{0 y}^{1}, A_{0 x}^{3}, A_{0 y}^{3}, \ldots, A_{0 x}^{6}, A_{0 y}^{6}\right\}^{\mathrm{T}}, \\
\left\{C_{m}^{0}\right\}=\left\{C_{0 x}, C_{0 y}\right\}^{\top}, \quad\left\{C_{s}^{0}\right\}=\{0,0,0,0,0,0,0,0,0,0\},
\end{gathered}
$$

Here the subscripts $m$ and $s$ denote master and slave, respectively. The [K] matrix can be partitioned as

$$
[K]=\left(\begin{array}{ll}
{\left[K_{m m}\right]^{(2 \times 2)}} & {\left[K_{m s}\right]^{(2 \times 10)}} \\
{\left[K_{s m}\right]^{(10 \times 2)}} & {\left[K_{s s}\right]^{(10 \times 10)}}
\end{array}\right) .
$$

The aim of this partition is to express the motion in terms of a reduced model in the master co-ordinates at the gap. From equation (7),

$$
\left[K_{m m}\right]\left\{A_{m}^{0}\right\}+\left[K_{m s}\right]\left\{A_{s}^{0}\right\}=\left\{C_{m}^{0}\right\}, \quad\left[K_{s m}\right]\left\{A_{m}^{0}\right\}+\left[K_{s s}\right]\left\{A_{s}^{0}\right\}=\left\{C_{s}^{0}\right\} .
$$

Combining equations (7) and (8) gives

$$
\left[\left[K_{m m}\right]-\left[K_{m s}\right]\left[K_{s s}\right]^{-1}\left[K_{s m}\right]\right]\left\{A_{m}^{0}\right\}=\left\{C_{m}^{0}\right\} .
$$

By setting

$$
[\bar{K}]=\left[K_{m m}\right]-\left[K_{m s}\right]\left[K_{s s}\right]^{-1}\left[K_{s m}\right] \quad(2 \times 2),
$$

equation (9) can be written as

$$
[\bar{K}]\left\{A_{m}^{u}\right\}=\left\{C_{m}^{0}\right\},
$$

where $[\bar{K}]$ is a reduced impedance matrix with constant terms since $\left\{C_{m}^{0}\right\}$ is a function of the displacement of node 2 at the bearing clearance as shown in equation (7), or

$$
\begin{aligned}
& C_{0 x}=C_{0 x}\left(A_{0 x}^{2}, A_{0 y}^{2}, A_{1 x}^{2}, A_{1 y}^{2}, \ldots, A_{N x}^{2}, A_{N y}^{2}, B_{N x}^{2}, B_{N y}^{2}\right), \\
& \mathrm{C}_{0 y}=\mathrm{C}_{0 y}\left(\mathrm{~A}_{0 \mathrm{x}}^{2}, \mathrm{~A}_{0 \mathrm{y}}^{2}, \mathrm{~A}_{1 \mathrm{x}}^{2}, \mathrm{~A}_{1 \mathrm{y}}^{2}, \ldots, \mathrm{A}_{\mathrm{Nx}}^{2}, \mathrm{~A}_{\mathrm{Ny}}^{2}, \mathrm{~B}_{\mathrm{Nx}}^{2}, \mathrm{~B}_{\mathrm{Ny}}^{2}\right) .
\end{aligned}
$$

It is noted that the constant coefficients $C_{0 x}, C_{0 y}$ in equation (5) are now expressed as functions of the unknowns $A_{0 x}^{2}, A_{0 y}^{2}, \ldots, B_{N x}^{2}, B_{N y}^{2}$. Equation (10) is an implicitly defined non-linear algebraic matrix to which the Newton-Raphson second order iterative method can be applied. To apply the Newton method, equation (10) is cast in an incremental form,

$$
[\bar{K}]\left\{\Delta A_{m}\right\}-\left\{\Delta C_{m}\right\}=\left\{C_{m}^{0}\right\}-[\bar{K}]\left\{A_{m}^{0}\right\} .
$$

Similarly, the coefficients associated with the cosine terms lead to

$$
-\left(\frac{n \omega}{\nu}\right)^{2}[M]\{A\}+\left(\frac{n \omega}{\nu}\right)[C]\{B\}+[K]\{A\}=\left\{C_{n}\right\}+\left\{\begin{array}{ll}
\left\{g_{c}\right\}, & \text { if } n=\nu \\
0, & \text { if } n \neq \nu
\end{array}\right\},
$$

where

$$
\begin{gathered}
(n=1,2, \ldots, N), \quad\{A\}=\left\{A_{n x}^{2}, A_{n y}^{2}, A_{n x}^{1}, A_{n y}^{1}, \ldots, A_{n y}^{6}\right\}^{\mathrm{T}}, \\
\{B\}=\left\{B_{n x}^{2}, B_{n y}^{2}, B_{n x}^{1}, B_{n y}^{1}, \ldots, B_{n y}^{6}\right\}^{\mathrm{T}}, \quad\left\{C_{n}\right\}=\left\{C_{n x}, C_{n y}, 0,0, \ldots, 0\right\}^{\mathrm{T}} .
\end{gathered}
$$

For the sine terms,

$$
-\left(\frac{n \omega}{\nu}\right)^{2}[M]\{B\}-\left(\frac{n \omega}{\nu}\right)[C]\{A\}+[K]\{B\}=\left\{D_{n}\right\}+\left\{\begin{array}{ll}
\left\{g_{s}\right\} & \text { if } n=\nu \\
0, & \text { if } n \neq \nu
\end{array}\right\},
$$


where

$$
(n=1,2, \ldots, N), \quad\left\{D_{n}\right\}=\left\{D_{n x}, D_{n y}, 0, \ldots, 0\right\}^{\mathrm{T}} .
$$

Combining equations (13) and (14) leads to

$$
[S]\{q\}=\{w\}+\{u\},
$$

where

$$
\begin{gathered}
\{q\}=\left\{A_{n x}^{2}, A_{n y}^{2}, B_{n x}^{2}, B_{n y}^{2}, A_{n x}^{1}, A_{n y}^{1}, B_{n x}^{1}, B_{n y}^{1}, \ldots, A_{n x}^{6}, A_{n y}^{6}, B_{n x}^{6}, B_{n y}^{6}\right\}^{\mathrm{T}}, \\
\{w\}=\left\{C_{n x}, C_{n y}, D_{n x}, D_{n y}, 0, \ldots, 0\right\}^{\mathrm{T}}, \quad\{u\}=\left\{g_{c}^{2}, 0,0, g_{s}^{2}, g_{c}^{1}, 0, \ldots, 0\right\}^{\mathrm{T}} .
\end{gathered}
$$

To reduce the size of equation (15), it is partitioned as

$$
\left(\begin{array}{ll}
{\left[S_{m m}\right]^{(4 \times 4)}} & {\left[S_{m s}\right]^{(4 \times 20)}} \\
{\left[S_{s m}\right]^{(20 \times 4)}} & {\left[S_{s s}\right]^{(20 \times 20)}}
\end{array}\right)\left(\begin{array}{c}
\left\{Q_{m}\right\} \\
\left\{Q_{s}\right\}
\end{array}\right)=\left(\begin{array}{c}
\left\{W_{m}\right\} \\
0
\end{array}\right)+\left(\begin{array}{c}
\left\{U_{m}\right\} \\
\left\{U_{s}\right\}
\end{array}\right)
$$

where

$$
\left\{Q_{m}\right\}=\left\{A_{n x}^{2}, A_{n y}^{2}, B_{n x}^{2}, B_{n y}^{2}\right\}^{\mathrm{T}}, \quad\left\{W_{m}\right\}=\left\{C_{n x}^{2}, C_{n y}^{2}, D_{n x}^{2}, D_{n y}^{2}\right\}^{\mathrm{T}} .
$$

From equation (16),

$$
\left[S_{m m}\right]\left\{Q_{m}\right\}+\left[S_{m s}\right]\left\{Q_{s}\right\}=\left\{W_{m}\right\}+\left\{U_{m}\right\}, \quad\left[S_{m s}\right]\left\{Q_{m}\right\}+\left[S_{s s}\right]\left\{Q_{s}\right\}=\left\{U_{s}\right\} . \quad(17 \mathrm{a}, \mathrm{b})
$$

Combining equations (17a) and (17b) gives

$$
\left[\left[S_{m m}\right]-\left[S_{m s}\right]\left[S_{s s}\right]^{-1}\left[S_{s m}\right]\right]\left\{Q_{m}\right\}=\left\{W_{m}\right\}+\left\{U_{m}\right\}-\left[S_{m s}\right]\left[S_{s s}\right]^{-1}\left\{U_{s}\right\} .
$$

With

$$
[\bar{S}]=\left[S_{m m}\right]-\left[S_{m s}\right]\left[S_{s s}\right]^{-1}\left[S_{s m}\right] \quad(4 \times 4), \quad\{\bar{U}\}=\left\{U_{m m}\right\}-\left[S_{m s}\right]\left[S_{s s}\right]^{-1}\left\{U_{s}\right\} \quad(4 \times 1),
$$

equation (18) can be expressed as

$$
[\bar{S}]\left\{Q_{m}\right\}=\left\{W_{m}\right\}+\{\bar{U}\},
$$

where $[\bar{S}]$ is a reduced impedance matrix with trigonometric terms. In an incremental form, equation (19) can be written as

$$
\left[\bar{S}_{n}\right]\left\{\Delta Q_{n}\right\}-\left\{\Delta W_{m n}\right\}=\left\{W_{m n}^{0}\right\}+\left\{\bar{U}_{n}\right\}-\left[\bar{S}_{n}\right]\left\{Q_{m n}^{0}\right\},
$$

where the subscript $n$ indicates the $n$th harmonic term, $n=1,2, \ldots, N$. Now, upon combining equations (12) and (20), the overall incremental equation of the system takes the form

$$
[T]\{\Delta r\}-\{\Delta v\}=\{z\},
$$

where

$$
\begin{aligned}
\{\Delta r\}= & \left\{\Delta A_{0 x}^{2}, \Delta A_{0 y}^{2}, \Delta A_{1 x}^{2}, \Delta A_{1 y}^{2}, \Delta B_{1 x}^{2}, \Delta B_{1 y}^{2}, \Delta A_{2 x}^{2}, \Delta A_{2 y}^{2}, \Delta B_{2 x}^{2}, \Delta B_{2 y}^{2}, \ldots,\right. \\
& \left.\Delta A_{N x}^{2}, \Delta A_{N y}^{2}, \Delta B_{N x}^{2}, \Delta B_{N y}^{2}\right\}^{\top} \\
\{\Delta v\}= & \left\{\Delta C_{0 x}, \Delta C_{0 y}, \Delta C_{1 x}, \Delta C_{1 y}, \Delta D_{1 x}, \Delta D_{1 y}, \Delta C_{2 x}, \Delta C_{2 y}, \Delta D_{2 x}, \Delta D_{2 y}, \ldots,\right. \\
& \left.\Delta C_{N x}, \Delta C_{N y}, \Delta C_{N x}, \Delta C_{N y}\right\}^{\top}
\end{aligned}
$$

and

$$
[T]=\left(\begin{array}{cccc}
{[\bar{K}]} & \cdots & \cdots & 0 \\
0 & {\left[\overline{S_{1}}\right]} & \cdots & 0 \\
\vdots & \vdots & \ddots & \vdots \\
0 & \cdots & \cdots & {\left[\overline{S_{N}}\right]}
\end{array}\right)(4 N+2,4 N+2)
$$


$[\bar{K}]$ is a $(2 \times 2)$ matrix, $\left[\bar{S}_{i}\right]$ is a $(4 \times 4)$ matrix and $i=1,2, \ldots, N$; also

$$
\begin{aligned}
\{z\}= & {\left[\left\{C_{m}^{0}\right\}-[\bar{K}]\left\{A_{m}^{0}\right\},\left\{W_{m 1}^{0}\right\}-\left\{\overline{U_{1}^{0}}\right\}-\left[\overline{S_{1}}\right]\left\{Q_{m 1}^{0}\right\},\left\{W_{m 2}^{0}\right\}-\left\{\overline{U_{2}^{0}}\right\}-\left[\overline{S_{2}}\right]\left\{Q_{m 2}^{0}\right\}, \ldots,\right.} \\
& \left.\left\{W_{m N}^{0}\right\}-\left\{\overline{U_{N}^{0}}\right\}-\left\{\overline{S_{N}}\right\}\left\{Q_{m N}^{0}\right\}\right]^{\mathrm{T}} .
\end{aligned}
$$

Since $C_{n x}, C_{n y}, D_{n x}$ and $D_{n y}$ depend on $A_{n x}^{2}, A_{n y}^{2}, B_{n, x}^{2}$, and $B_{n y}^{2}$, the increments can be expanded as

$$
\begin{aligned}
\Delta C_{n x}= & \frac{\partial C_{n x}}{\partial A_{0 x}^{2}} \Delta A_{0 x}^{2}+\frac{\partial C_{n x}}{\partial A_{0 y}^{2}} \Delta A_{0 y}^{2} \\
& +\sum_{l=1}^{N}\left(\frac{\partial C_{n x}}{\partial A_{l x}^{2}} \Delta A_{l x}^{2}+\frac{\partial C_{n x}}{\partial A_{l y}^{2}} \Delta A_{l y}^{2}+\frac{\partial C_{n x}}{\partial B_{l x}^{2}} \Delta B_{l x}^{2}+\frac{\partial C_{n x}}{\partial B_{l y}^{2}} \Delta B_{l y}^{2}\right), \\
\Delta D_{n x}= & \frac{\partial D_{n x}}{\partial A_{0 x}^{2}} \Delta A_{0 x}^{2}+\frac{\partial D_{n x}}{\partial A_{0 y}^{2}} \Delta A_{0 y}^{2} \\
& +\sum_{l=1}^{N}\left(\frac{\partial D_{n x}}{\partial A_{l x}^{2}} \Delta A_{l x}^{2}+\frac{\partial D_{n x}}{\partial A_{l y}^{2}} \Delta A_{l y}^{2}+\frac{\partial D_{n x}}{\partial B_{l x}^{2}} \Delta B_{l x}^{2}+\frac{\partial D_{n x}}{\partial B_{l y}^{2}} \Delta B_{l y}^{2}\right),
\end{aligned}
$$

where $n$ denotes the $n$th harmonic mode. A similar expression can be written for $\Delta C_{n y}$ and $\Delta D_{n y}$ for the $y$ direction. From equation (21), $\{\Delta v\}$ is written with partial derivatives of $\{\Delta r\}$ terms since $\{\Delta v\}$ is a function of $\{\Delta r\}$, as shown by equation (22). The partial derivative terms yield the Jacobian matrix, so one can finally obtain the equation

$$
([T]+[P])\{\Delta r\}=\{z\},
$$

where

$$
[P]=\left(\begin{array}{ccccc}
\partial C_{0 x} / \partial A_{0 x}^{2} & \partial C_{0 x} / \partial A_{0 y}^{2} & \partial C_{0 x} / \partial A_{1 x}^{2} & \cdots & \partial C_{0 x} / \partial B_{N_{y}}^{2} \\
\partial C_{0 y} / \partial A_{0 x}^{2} & \partial C_{0 y} / \partial A_{0 y}^{2} & \partial C_{0 y} / \partial A_{1 x}^{2} & \cdots & \partial C_{0 y} / \partial B_{N y}^{2} \\
\vdots & \vdots & \vdots & \ddots & \vdots \\
\partial D_{N y} / \partial A_{0 x}^{2} & \partial D_{N y} / \partial A_{0 y}^{2} & \partial D_{N_{y}} / \partial A_{1 x}^{2} & \cdots & \partial D_{n y} / \partial B_{N y}^{2}
\end{array}\right)
$$

Here the superscripts denote the degree of freedom number and a subscript denotes the harmonic term in $x$ or $y$ direction. The total dimension of the unknown vector $\{\Delta r\}$ is $4 N+2$. This $[P]$ matrix has to be found by using numerical differentiation with discrete and inverse discrete FFT, and it has to be updated at each iteration until the Euclidean norm of the unknown vector $\{\Delta r\}$ converges. More details on the calculation procedure are given in the paper by Choi and Noah [4].

\section{NUMERICAL RESULTS AND DISCUSSION}

The methods as formulated in the previous section have been applied to the rotor shown in Figure 1. A density of $7835 \mathrm{~kg} / \mathrm{m}^{3}$ and an elastic modulus of $2.07 \times 10^{11} \mathrm{~N} / \mathrm{m}^{2}$ were used for the shaft. The geometric data of its three elements is listed in Table 1.

The first three critical speeds of the linear rotor in absence of gyroscopic terms were computed by using the harmonic balance method. All the critical speeds are compared with results for the eigenvalues as computed by the commercial package (IMSL) subroutine EIGZF. The results are identical and a Bode plot is shown in Figure 2. A comparison of the accuracy of the direct numerical integration (fourth order Runge-Kutta) and the harmonic balance method (HBM) is shown in Figure 3. The steady state response obtained by using numerical integration was calculated by using equation (1). For the 
TABLE 1

Rotor configuration and physical parameters

\begin{tabular}{ccc}
\hline & Item & Value \\
\hline$E$ & Young's modulus & $2.07 \times 10^{11} \mathrm{~N} / \mathrm{m}^{2}$ \\
$m_{1}$ & Mass of first disk & $1.18 \mathrm{~kg}$ \\
$m_{2}$ & Mass of second disk & $5.76 \mathrm{~kg}$ \\
O.D. & Shaft outer diameter & $0.054 \mathrm{~m}$ \\
I.D. & Shaft inner diameter & $0.019 \mathrm{~m}$ \\
$L_{1}$ & 1st element length & $0.091 \mathrm{~m}$ \\
$L_{2}$ & 2nd element length & $0.116 \mathrm{~m}$ \\
$L_{3}$ & 3rd element length & $0.1 \mathrm{~m}$ \\
$K_{B}$ & Non-linear spring stiffness & $7.7 \times 10^{7} \mathrm{~N} / \mathrm{m}$ \\
$J_{d 1}$ & 1st disk diametrical moment of inertia & $0.056 \mathrm{~kg} \mathrm{~m}$ \\
$J_{d 2}$ & 2nd disk diametrical moment of inertia & $0.114 \mathrm{~kg} \mathrm{~m}$ \\
\hline
\end{tabular}
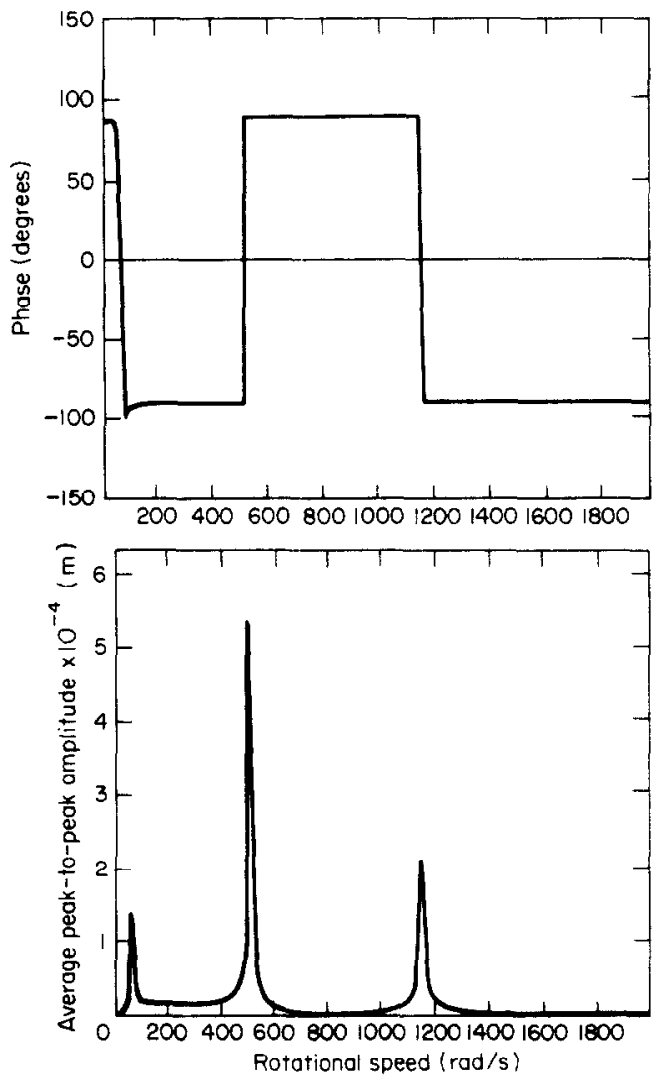

Figure 2. Bode plot of the radial response at the bearing with clearance (no clearance, no gyroscopic effect). 


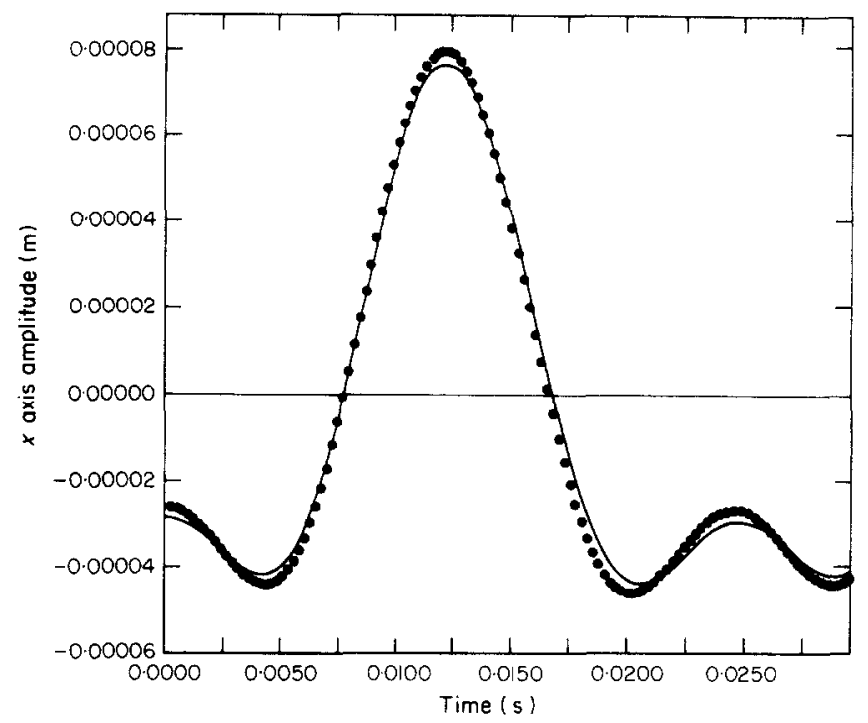

Figure 3. Comparison of non-linear rotor response at the bearing with numerical integration at rotational speed $=1020 \mathrm{rad} / \mathrm{s} ;$ radial clearance $=1 \cdot 27 \times 10^{-5} \mathrm{~m}$, mass eccentricity $=2.54 \times 10^{-5} \mathrm{~m}$, side force $=10 \mathrm{~N}$. $\longrightarrow$, HBM; $\bigcirc$ numerical integration.

application of the HBM method in a numerical procedure, the first four harmonic coefficients were used, which is the most efficient in calculating subharmonics [4]. As shown in Figure 3, a dominant $1 / 2$ order subharmonic response obtained by using the HBM method demonstrates good agreement with the direct numerical integration results, not only for the waveforms for displacement but also for phase. The slight difference (within $5 \%$ error) is due to ignoring the higher harmonic terms in the HBM method. As more harmonics are added, the HBM method results converge to those obtained by using numerical integration. The calculation time of the HBM method is superior to that of the numerical integration method. For most simulation cases, the numerical integration method takes several hundred times longer than the HBM method to yield the steady state response for a typical multi-degree-of-freedom rotor model such as the one utilized herein. However, better computational performance of the numerical integration can be achieved if (i) the integration step size is taken larger, (ii) the system damping is large, and (iii) the system has a small number of degrees of freedom.

When the rotor is subjected to imbalance forces, damaging $1 / 2$ order or the lower subharmonics near twice or $n$ times the natural frequency appear in the presence of the deadband clearance and a side (radial) force due to gravity and misalignment. To demonstrate the occurrence of significant subharmonic response, the damping was set to a very small value. The results presented in Figure 4 and subsequent figures were obtained with account taken of the gyroscopic moments due to rotation of the two disks. As shown in Figure 4, the 1/2 order subharmonic response occurs near twice the second natural frequency and is dominant. This can cause a damaging effect over the operating speed. The shaft center may trace either circular or elliptical paths under the harmonic forces. However, in cases of subharmonic response, the paths will be of more complicated forms. In Figure 5, a typical 1/2 order subharmonic response with harmonic shaft trajectory is shown. The existence of the $1 / 3$ order and the lower order subharmonic components 


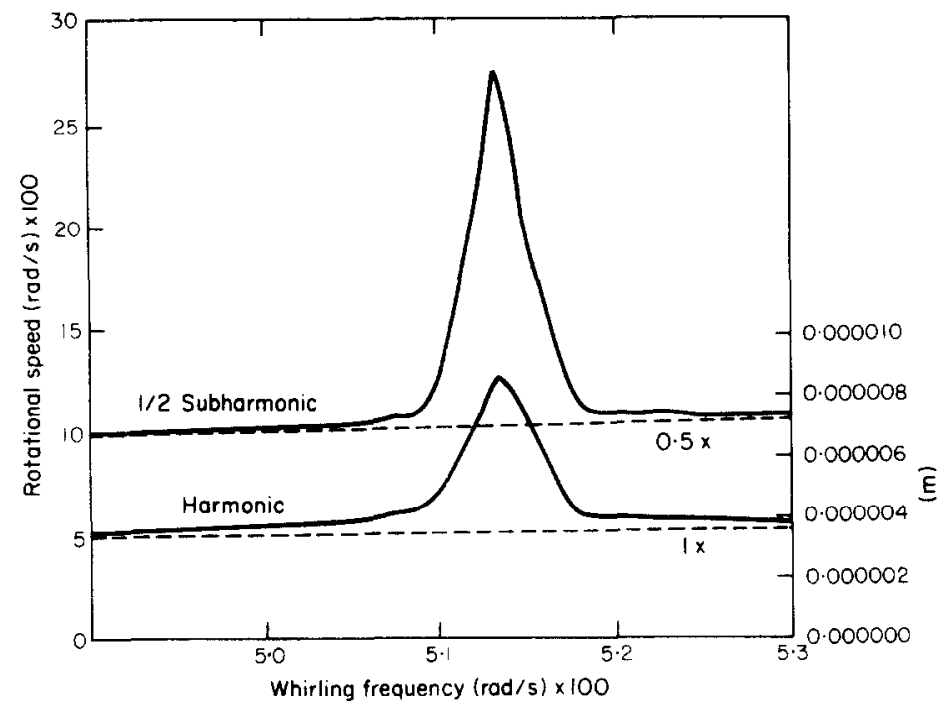

Figure 4. Comparison between subharmonic and harmonic responses at the bearing; radial clearance = $1.27 \times 10^{-5} \mathrm{~m}$, mass eccentricity $=1.27 \times 10^{-5} \mathrm{~m}$, side force $=44.5 \mathrm{~N}$.

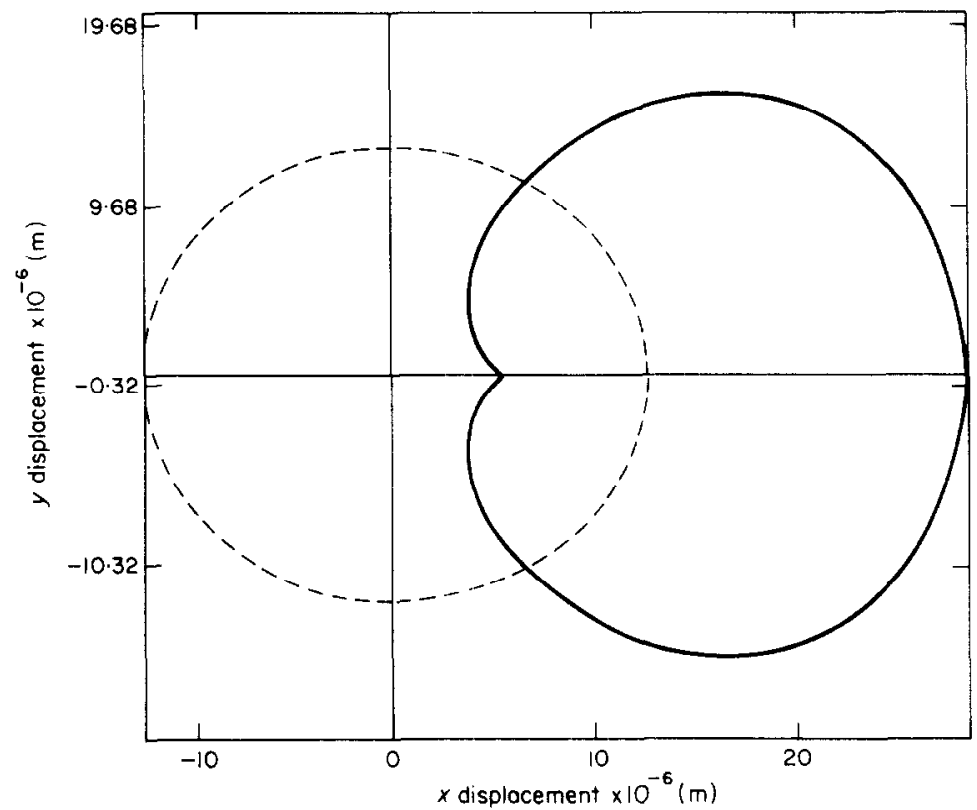

Figure 5. Orbit of the subharmonic response at the bearing with rotational speed $=1020 \mathrm{rad} / \mathrm{s}$; radial clearance $=1.27 \times 10^{-5} \mathrm{~m}$, mass eccentricity $=1.4 \times 10^{-5} \mathrm{~m}$, side force $=22.25 \mathrm{~N}$.

were examined for various values of the side force, gap size and eccentricity, near the natural frequencies. As well as the $1 / 2$ order subharmonic response, the $1 / 3$ order subharmonic response can be as dangerous since the peak to peak precession amplitude of a $1 / 3$ order subharmonic can be very large in some extreme cases, as shown in Figure 6. The figure shows that the dominant response is the $1 / 3$ order subharmonic component. A $1 / 4$ order subharmonic response can also exist under certain conditions, as shown in Figure 7 . These results confirm that high-speed rotating machinery with asymmetry in 


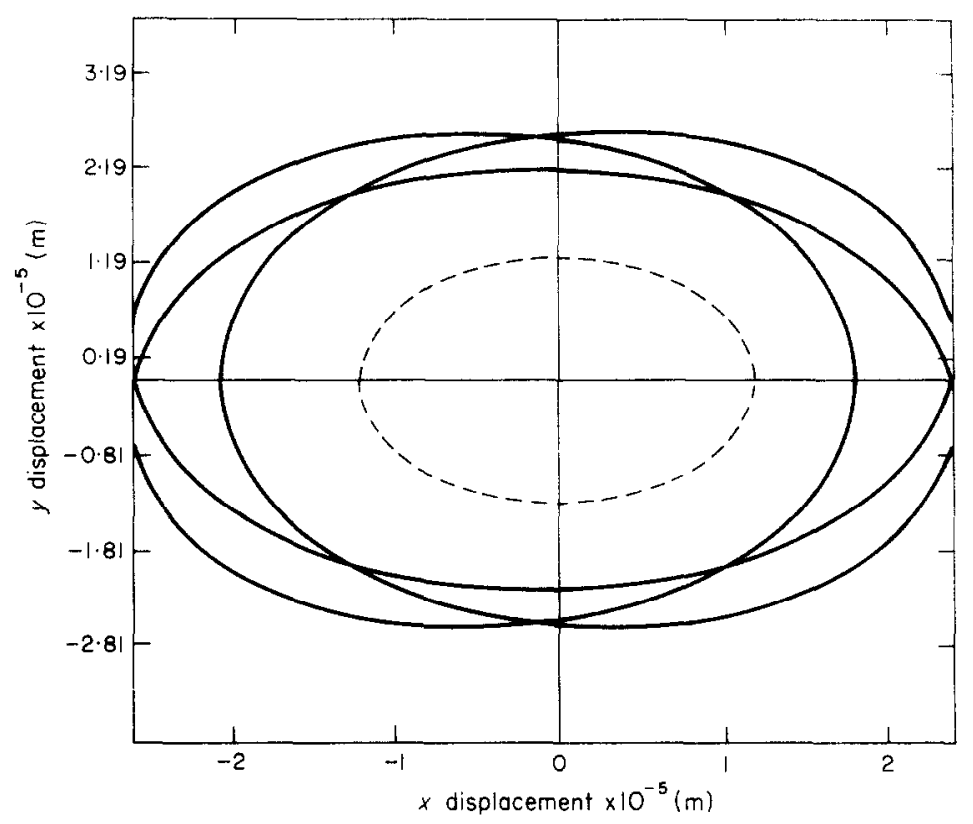

Figure 6. Orbit of the subharmonic response at the bearing with rotational speed $=3360 \mathrm{rad} / \mathrm{s}$; radial clearance $=1.27 \times 10^{-5} \mathrm{~m}$, mass eccentricity $=5.08 \times 10^{-5} \mathrm{~m}$, side force $=44.5 \mathrm{~N}$.

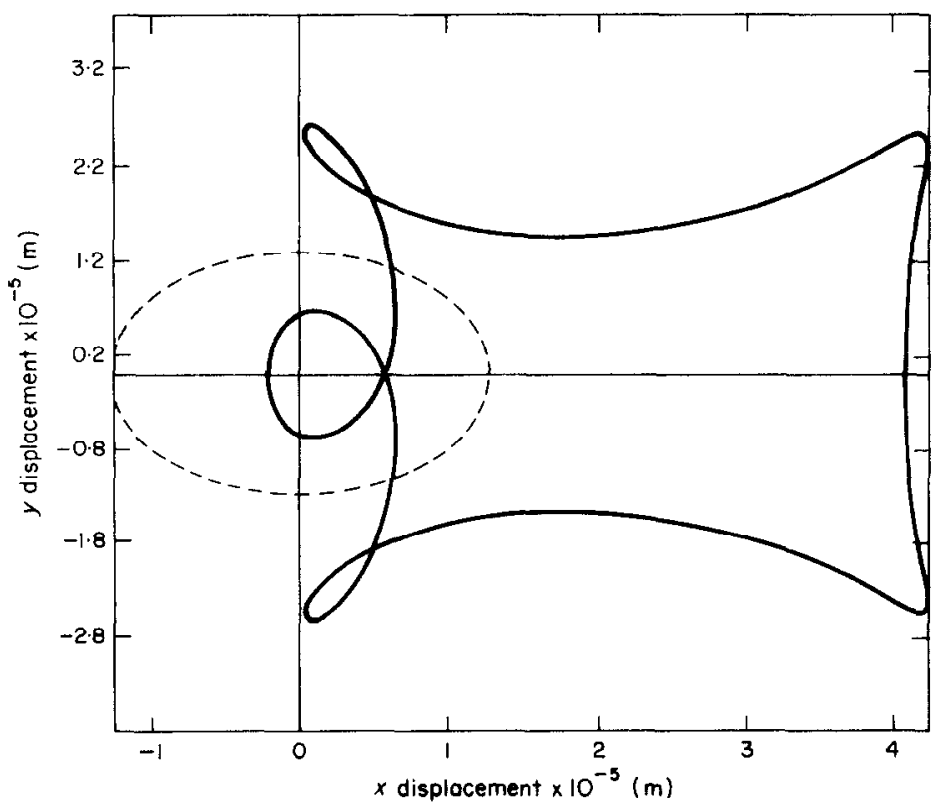

Figure 7. Orbit of the subharmonic response at the bearing with rotational speed $=1960 \mathrm{rad} / \mathrm{s}$; radial clearance $=1.27 \times 10^{-5} \mathrm{~m}$, mass eccentricity $=0.89 \times 10^{-5} \mathrm{~m}$, side force $=22.25 \mathrm{~N}$. 
the bearing supports might have a lower order subharmonic vibration response [14]. In a recent paper submitted by the present authors [15], the same whirling shapes for a model presented in reference [14] were obtained by using the harmonic balance method.

The effects of the side force, eccentricity and gap size play a significant role in the occurrence of dangerous subharmonics as mentioned above. In earlier work, Choi and Noah [4] showed that dangerous subharmonic responses could develop for a rotor system for a certain range of the side force magnitude. The effects of the side force and mass eccentricity were investigated, and the results are presented in Figures 8,9 and 10. In Figure 8 , with gap sizes smaller than $2.54 \times 10^{-5} \mathrm{~m}$, the eccentricity has the dominant effect on the subharmonic response. The figure shows that for a certain range of side force magnitudes (between $50 \mathrm{~N}$ and $250 \mathrm{~N}$ ), the subharmonic response can exist. In Figure 9 are shown the side forces and eccentricities at which the maximum $1 / 2$ order subharmonic component occurs. The figure shows that for a given bearing clearance higher side force is required to produce significant subharmonic motion when the eccentricity is small (lower than $5 \times 10^{5} \mathrm{~m}$ ). However, for side forces higher than some value, the eccentricity has no effect on the occurrence of subharmonics, since the bearing outer race will be already in contact with the housing. This trend is the same for all gap sizes.

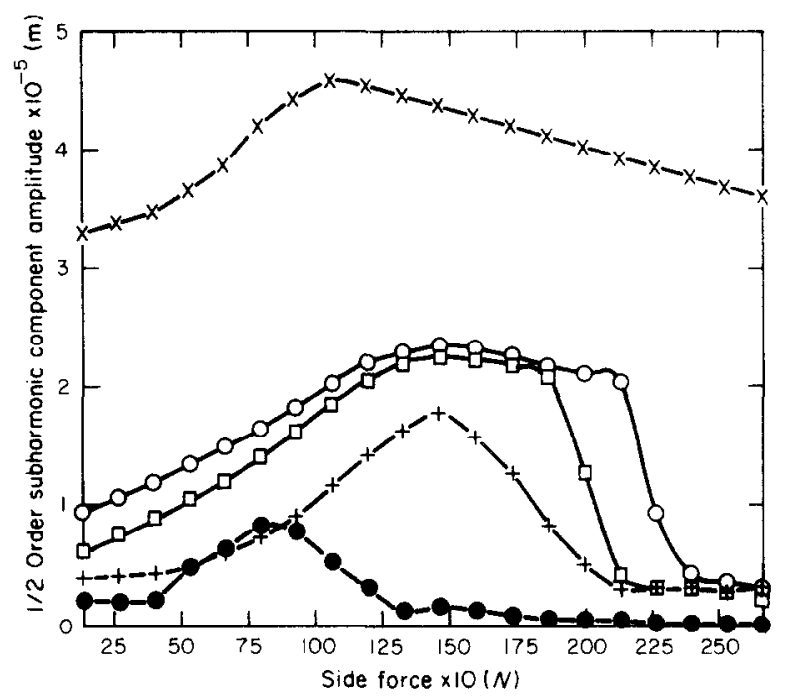

Figure 8. Effect of side force and eccentricity at the bearing with frequency $=1180 \mathrm{rad} / \mathrm{s} ;$ radial clearance $=$ $2.54 \times 10^{-5} \mathrm{~m}$. Eccentricity $\times 10^{-5}(\mathrm{~m}):-\square_{-2} \cdot 54 ;-+, 5 \cdot 08 ;-\square-, 7 \cdot 82 ;-0-, 10 \cdot 18 ;-\times-, 12 \cdot 70$.

The effects of the side force and gap size were studied and the results are shown in Figure 10. This figure shows values of the side forces and gap sizes at which the maximum $1 / 2$ order subharmonic response occurs. The figure also shows that the gap size is significant in determining whether or not large subharmonics occur. This figure shows that if the gap size becomes larger, more side force is required for the occurrence of the subharmonics. This is because for larger gap sizes more side force is needed to keep intermittent contacting between bearing and housing. Therefore, a well balanced rotor will not have the damaging subharmonic response (or subsynchronous motions) even if 


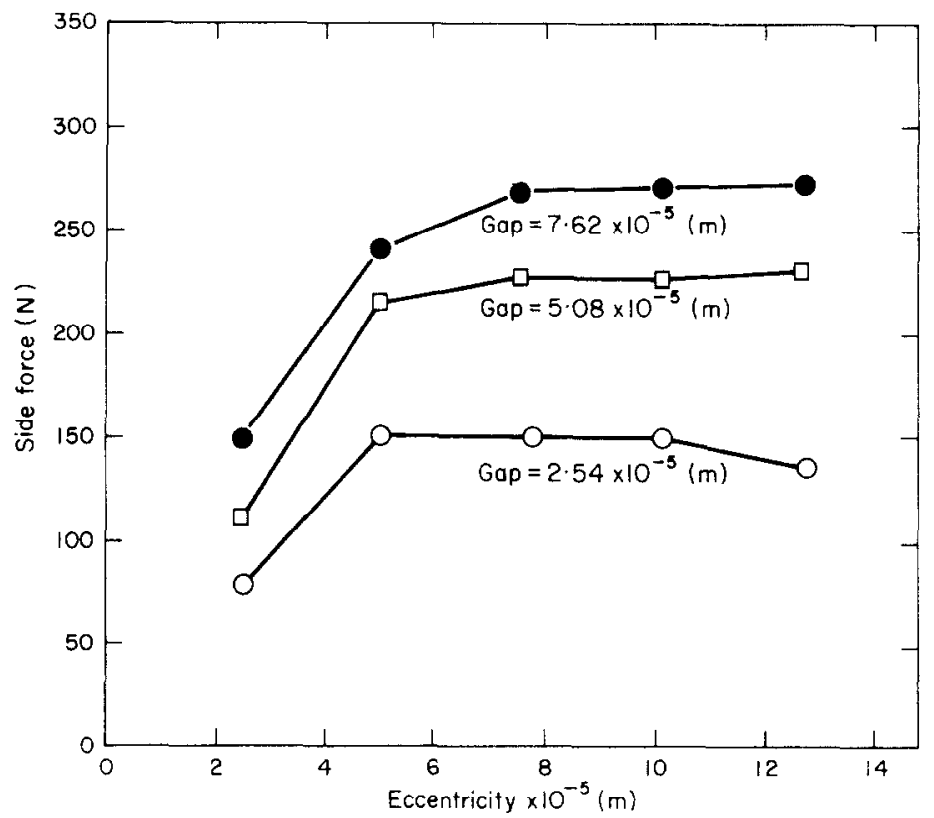

Figure 9. Side force and mass eccentricity for peak subharmonic component at $1180 \mathrm{rad} / \mathrm{s}$.

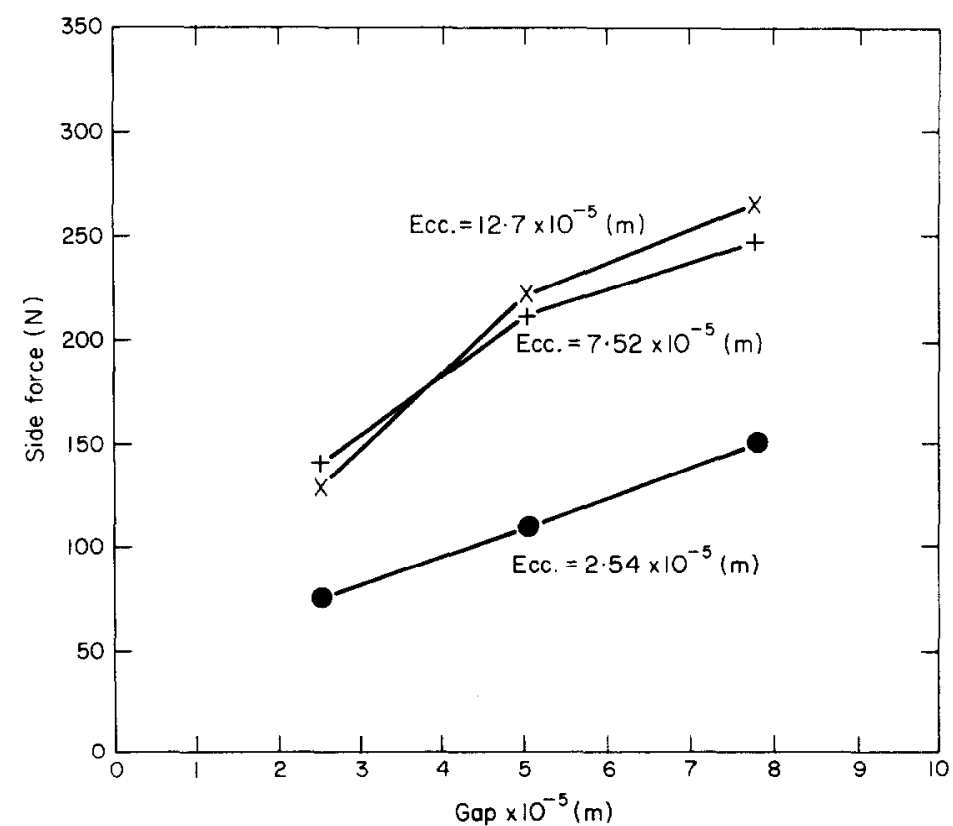

Figure 10. Side force and radial clearance (gap size) for peak subharmonic component at $1180 \mathrm{rad} / \mathrm{s}$.

there exists a deadband between bearing outer race and housing. If the rotor is poorly balanced, a possible procedure to avoid a subharmonic response is to decrease the side force or the gap size. 


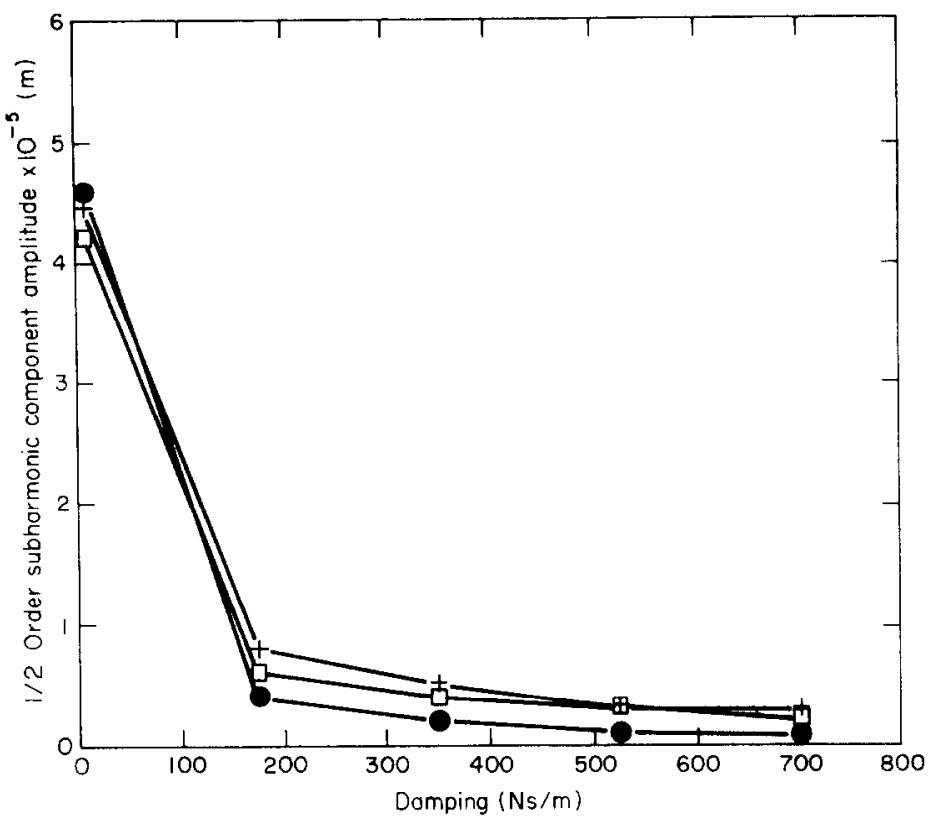

Figure 11. Effect of damping at bearing on the subharmonic response at $1180 \mathrm{rad} / \mathrm{s}$; radial clearance $=$ $2.54 \times 10^{-5} \mathrm{~m}$ and mass eccentricity $=12.7 \times 10^{-5} \mathrm{~m}$. Side force $(\mathrm{N}):--, 10 ;-+-, 150 ; \square \square-, 200$.

The effect of damping at the bearing was investigated and the result is shown in Figure 11. The figure shows that the increase in damping results in a smaller subharmonic response, with a more significant effect on reducing or eliminating the subharmonics being observed for values of the viscous damping coefficient larger than $175 \mathrm{~N} \mathrm{~s} / \mathrm{m}$. The side force does not affect this tendency.

\section{CONCLUSIONS}

An effective numerical algorithm for determining the steady state forced vibration of a strongly non-linear multi-degree-of-freedom rotor system has been developed. The method provides an accurate (with $\leqslant 5 \%$ error) and efficient means for determining the non-linear effects on rotor dynamic behavior. In addition, the complicated non-linear steady state periodic motions of multi-degree-of-freedom rotor systems can readily be studied by using the present method. Even though the method can accommodate any number of retained harmonics, there is a trade-off between level of accuracy and computational cost. In general, four harmonics were found to be adequate for good accuracy at a reasonable cost. Another advantage of the harmonic balance method is that it is capable of providing the unstable as well as the stable solutions. This is necessary if the global dynamic behavior of the system is to be determined. This includes bifurcation analysis and possible occurrence of chaos.

The newly developed algorithm was applied to a typical multi-disk rotor/bearing system. Dangerous subharmonic resonances may occur in the presence of bearing clearances, mass eccentricities and side forces due to gravity or misalignments. The results show that the ranges of the above three parameters are mutually related in determining the subharmonic occurrence. The side force and size of radial clearance within certain ranges can 
induce significant subharmonic responses. These results can be explained by the contacting motion between bearing outer race and housing which might render the system highly non-linear. Once the stiffness starts changing due to the contact motion, the stiffness variation is not affected by increasing the level of the side forces. These characteristics of stiffness variation due to the bearing-clearance effects well agree with other previous experimental results [2] and analytical results $[6,13]$.

\section{ACKNOWLEDGMENTS}

This work was carried out as part of a research project supported by NASA, Marshall Flight Center under contract No. NAS8-36293. The authors are grateful to Thomas Fox, the technical monitor, for his enthusiastic support and interest.

\section{REFERENCES}

1. R. F. BEATTY 1985 American Society of Mechanical Engineers, Journal of Vibration, Acoustics, Stress, And Reliability in Design 107, 151-160. Differentiating rotor response due to radial rubbing.

2. D. BENTLY 1974 ASME Paper No. 74-PET-16, Petroleum Mechanical Engineering Conference, Dallas, Texas, September. Forced subrotative speed dynamic action of rotating machinery.

3. F. F. EHRICH 1966 ASME Paper No. 66-MD-1 Design Engineering Conference and Show, Chicago, Illinois, 9-12 May. Subharmonic vibrations of rotors in bearing clearance.

4. Y. S. CHOI and S. T. NOAH 1987 American Society of Mechanical Engineers, Journal of Vibration, Acoustics, Stress, And Reliability in Design 109, 255-261. Nonlinear steady-state response of a rotor-support system.

5. T. T. YAMAMOTO 1954 Memoirs of the Faculty of Engineering, Nagoya (Japan) University 6. On critical speeds of a shaft.

6. D. W. CHILDS 1982 American Society of Mechanical Engineers, Journal of Energy and Power 104, 533-541. Fractional-frequency rotor motion due to nonsymmetric clearance effects.

7. S. SAITO 1985 American Society of Mechanical Engineers Paper No. 85-DET-33. Calculation of nonlinear unbalance response of horizontal Jeffcott rotors supported by ball bearings with radial clearances.

8. C. NATARAJ and H. D. Nelson 1987 American Society of Mechanical Engineers Design Conference, October. Periodic solutions in rotor dynamic systems with nonlinear supports: a general approach.

9. R. J. CipRA and J. J. UiCKeR JR. 1981 American Society of Mechanical Engineers, Journal of Mechanical Design 103, 849-865. On the dynamic simulation of large nonlinear mechanical systems, part 1: an overview of the simulation technique. Substructuring and frequency domain considerations.

10. R. D. NEILSON and A. D. S. BARR 1987 Seventh World Congress on the Theory of Machines and Mechanisms, Seville, 1799-1803. Spectral features of the response of a rigid rotor mounted on discontinuously nonlinear supports.

11. S. T. NOAH 1984 Final Report on NASA Contract NAS8-34505, Texas A\&M University, September. Rotordynamic analysis of the SSME turbopumps using reduced models.

12. U. J. FAN and S. T. NOAH American Institute of Aeronautics and Astronautics Journal of Propulsion and Power 47, 53-73. Vibration analysis of rotor systems using reduced subsystem models.

13. A. N. JEAN and H. D. NELSON 1989 American Society of Mechanical Engineers, Vibrations Conference, Montreal, September. Periodic response investigation of large order nonlinear rotordynamic systems using collocation.

14. F. F. EHRICH 1988 American Society of Mechanical Engineers, Journal of Vibration, Acoustics, Stress, And Reliability in Design 110, 9-16. High order subharmonic response of high speed rotors in bearing clearance.

15. Y. B. KIM and S. T. NOAH 1990 Nonlinear Dynamics (to appear). Bifurcation analysis for a modified Jeffcott rotor with a bearing clearance. 


\section{APPENDIX: NOMENCLATURE}

$\left\{A_{m}^{0}\right\} \quad$ master degree of cosine coefficient of steady state solution $(2 \times 1)$

$\left\{\Delta \boldsymbol{A}_{m}\right\} \quad$ incremental vector of $\left\{\boldsymbol{A}_{m}^{0}\right\}$

$A_{n x}^{i}, A_{n y}^{i} \quad n$th cosine coefficient of the steady state solution in $x, y$ direction at $i$ th node

$\left\{\boldsymbol{A}_{s}^{O}\right\} \quad$ slave degree of cosine coefficient of steady state solution $(2 \times 1)$

$B_{n,}^{i}, B_{n y}^{i} \quad n$th sine coefficient of the steady state solution in $x, y$ direction at $i$ th node

$[C]$ damping matrix $(12 \times 12)$

$\left\{C_{n}\right\} \quad$ constant cosine coefficient vector of non-linear force $(12 \times 1)$

$C_{n \times}, C_{n y} \quad n$th cosine coefficient of non-linear force in $x, y$ direction

$\left\{C_{m}^{0}\right\}$ master degree of non-linear force vector with cosine coefficient $(2 \times 1)$

$\left\{C^{\prime}\right\}$ slave degree of non-linear force vector with cosine coefficient $(10 \times 1)$

$\left\{D_{n}\right\} \quad$ constant sine coefticient vector of non-linear force $(12 \times 1)$

$D_{n x}, D_{n y} \quad n$th sine coefficient of non-linear force in $x, y$ direction

$[K] \quad$ stiffness matrix $(12 \times 12)$

$K_{\underline{B}} \quad$ bearing stiffness

$[\bar{K}] \quad$ reduced impedance matrix with constant terms $(2 \times 2)$

[M] mass matrix $(12 \times 12)$

$\left\{Q_{m}\right\} \quad$ master degree of trigonometric coefficient vector of steady state solution $(4 \times 1)$

$\left\{Q_{m n}^{0}\right\} \quad$ master degree of trigonometric coefficient vector of steady state solution with $n$th harmonic mode $(4 \times 1)$

$\left\{\Delta Q_{n}\right\} \quad$ incremental vector of $\left\{Q_{m n}^{0}\right\}$

$\left\{Q_{s}\right\} \quad$ slave degree of trigonometric coefficient vector $(20 \times 1)$

$\{R\} \quad$ displacement vector $(12 \times 1)$

$R_{i x}, R_{i y} \quad x, y$ rectlinear displacement at $i$ th node

$[S] \quad$ total trigonometric coefficient matrix $(24 \times 24)$

$[\bar{S}] \quad$ reduced impedance matrix with trigonometric terms $(4 \times 4)$

$\left[\overline{S_{n}}\right] \quad$ reduced impedance matrix with trigonometric terms of $n$th harmonic mode $(4 \times 4)$

$[T] \quad$ overall coefficient matrix $(4 N+2,4 N+2)$

$\{\bar{U}\} \quad$ equivalent trigonometric force vector $(4 \times 1)$

$\left\{\boldsymbol{W}_{m}\right\}$ master degree of trigonometric coefficient vector of non-linear force $(20 \times 1)$

$\left\{\Delta W_{m n}\right\} \quad$ incremental vector of $\left\{W_{m n}^{0}\right\}$ master degree of trigonometric vector of non-linear force with $n$th harmonic mode $(4 \times 1)$

$\left\{f_{x}, f_{y}\right\}$

$\{g(t)\}$

$\left\{g_{c}, g_{s}\right\}$

$\{q\}$

$\{\Delta r\}$

$\{u\}$

$\{\Delta v\}$

$\{w\}$

$\{z\}$

$\beta_{i x}, \beta_{i y}$

Non-linear restoring vector $(12 \times 1)$

non-linear restoring force vector in $x, y$ direction

imbalance force vector $(12 \times 1)$

imbalance force vector of cosine, sine terms $(12 \times 1)$

constant trigonometric coefficient vector of steady state solution $(24 \times 1)$

overall incremental coefficient vector $(4 N+2,1)$

total imbalance force vector $(24 \times 1)$

overall incremental coefficient vector of non-linear force $(24 \times 1)$

constant trigonometric coefficient vector of non-linear force $(24 \times 1)$

overall force vector $(4 N+2,1)$

$\delta$ radial clearance

Subscripts

$B$ bearing side

$N \quad$ total retained harmonic modes

$m$ master

$n \quad n$th harmonic mode

$s \quad$ slave

$x \quad x$ direction

$y \quad y$ direction

Superscript

$i \quad i$ th node 